\title{
Prevalence of Myasthenia progression in hepatic encephalopathy syndrome
}

\author{
Israa Burhan Raoof ${ }^{1, *}$, Mayssaa E. Abdalah ${ }^{1}$ \\ Department of Clinical Laboratory Science, College of Pharmacy, Mustansiriyah University, Baghdad- Iraq
}

Articles Information

Received:

$10,10,2019$

Accepted:

29, 12,2019

Published:

01, March, 2020

\section{Keywords:}

Myasthenia progression

Hepatic encephalopathy

Cirrhosis

\begin{abstract}
Hepatic encephalopathy (HE) is a neurological and psychological syndrome related to acute and chronic liver failure especially during cirrhosis, it is associated with over production of ammonia in gastrointestinal tract with failure excretion by the liver in addition to increased free radical and bacterial transmission have played an important role. A symptom of disease includes coma, ophthalmia, irregular sleeping, stress and difficult breathing. It may be develop to intestinal bleeding; reduced blood pressure and electrolyte disturbances. Myasthenia is early symptoms of muscle dystrophies and prevalence of antibodies against acetylcholine receptors, nicotine receptors, tyrosine kinase and low-density lipoprotein proteins thus severely weakening muscles and skeletons have been demonstrated, it is playing important role in myasthenia progressing especially after five years of liver antibodies detection. Recently, the treatment is mainly depending on the reduced intestinal ammonia with non-absorbent disacchaᄀrides from intestine and MRI should be used to follow up in addition to newer immunosuppressive drugs such as Rifaxamin, an inhibitor of interleukins and steroids is very important to treat this disease.
\end{abstract}

DOI: $10.22401 /$ ANJS.23.1.02

* Corresponding author: israaburhan@uomustansiriyah.edu.iq

Hepatic encephalopathy (HE) is a brain dysfunction associated with liver failure or progression of neurological disorders. Clinically, symptoms of the disease include coma and weakness, according to recent studies Jaundice and other liver diseases can be particularly related to mood and behavioral disorders [1]. HE can occurs in acute or chronic liver failure [2]. Cirrhotic patients diagnosed with abnormal of electrical brain and neuropsychological tests and the spectrum of cognitive neuropathy in cirrhosis is a continuum rather than a conclusive one[3]. Recently it is suggested that neurotoxins effect on neurotransmitters by impaired brain energy and neurological responses $[4,5]$, lactate is accumulated in the extracellular cerebral cortex but decrease level of lactate within the cells [6]. Other characteristic physiological factors include free radicals and imbalances in the permeability of the blood-brain barrier [7].

In figure 1 (A) Orinithin phenyl acetate produce glutamine from L-ornithine in blue color (B). In the kidney, Glutamine converted to phenyl-acetyl glutamine followed by its ultimate secretion (C)
Ammonia is converted to glutamine leading to low levels of systemic and cerebral ammonia while hyperammonium destroyed the astrocytes and neural cell, Rifaxamin and Lactulose are effectively drugs to reduce the production of bacterial ammonia [7] Hyperammonemia is a play a major role in the hepatic encephalopathy with stimulated of inflammation, cytokines and neurotransmission therefore in cirrhotic patients, microglial and astrocytes cells is induced tumour necrosis factor (TNF) to alter the permeability of blood brain barrier and increased ammonia diffusion, in addition to depleting Zinc concentration playing role to hyperammonemia complication while increased manganese which accumulated in the basal ganglia [8]. In line with this idea, a study shows that the variable tumour growth factor (TGF) increases the permeability of the blood barrier for ammonia [9]. The digestive system is the main sources of ammonia; it was produced from glutamine or nitrogen degradation by colonic bacteria especially in the gastrointestinal bleeding [10]. The etiology of hepatic encephalopathy is showed in figure 2 [11]. 


\section{Al-Nahrain Journal of Science}

\section{ANJS, Vol.23 (1), March, 2020, pp. 7-12}

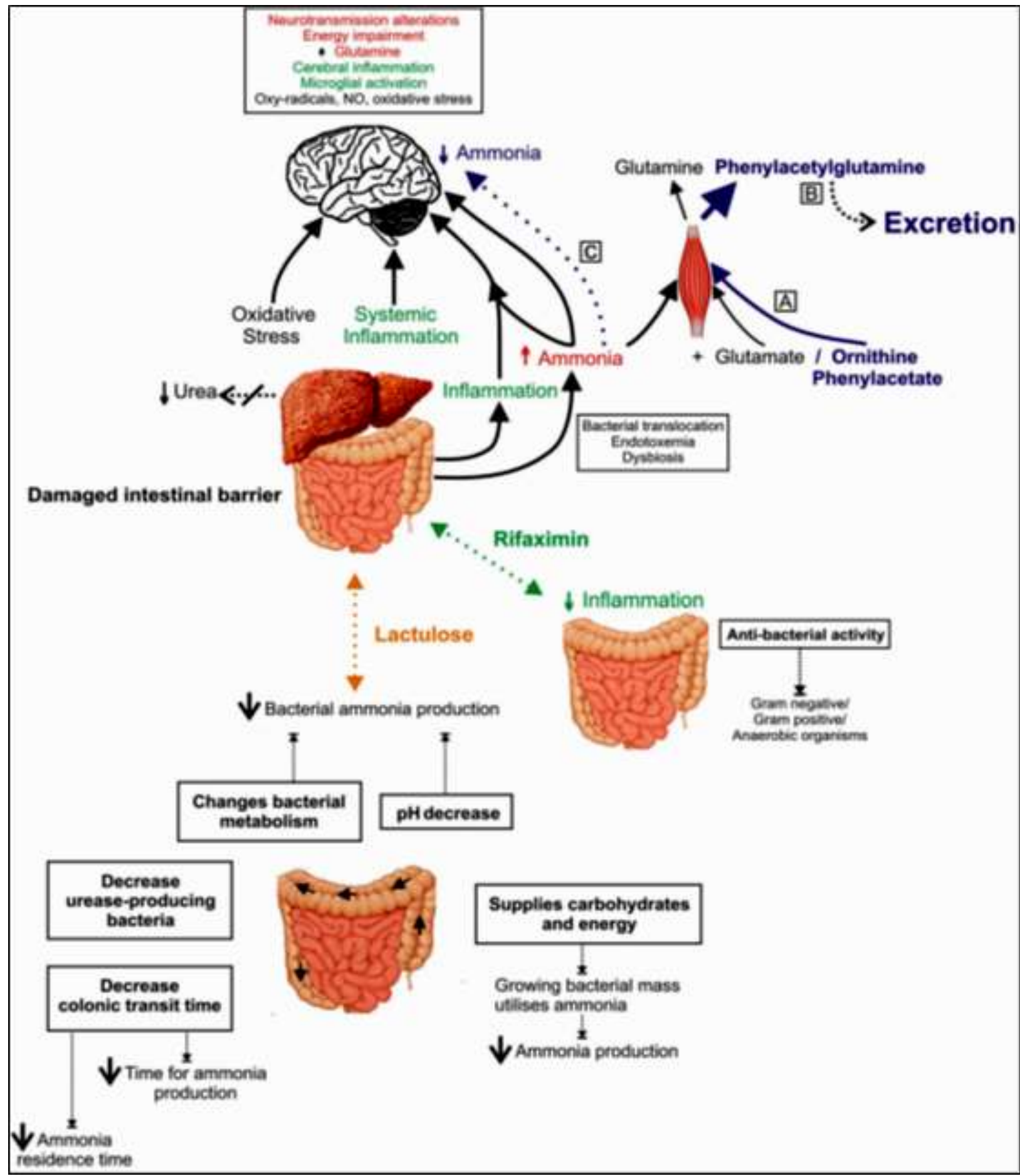

Figure 1. Hepatic encephalopathy mechanisms [7]. 


\section{Al-Nahrain Journal of Science}

ANJS, Vol.23 (1), March, 2020, pp. 7-12

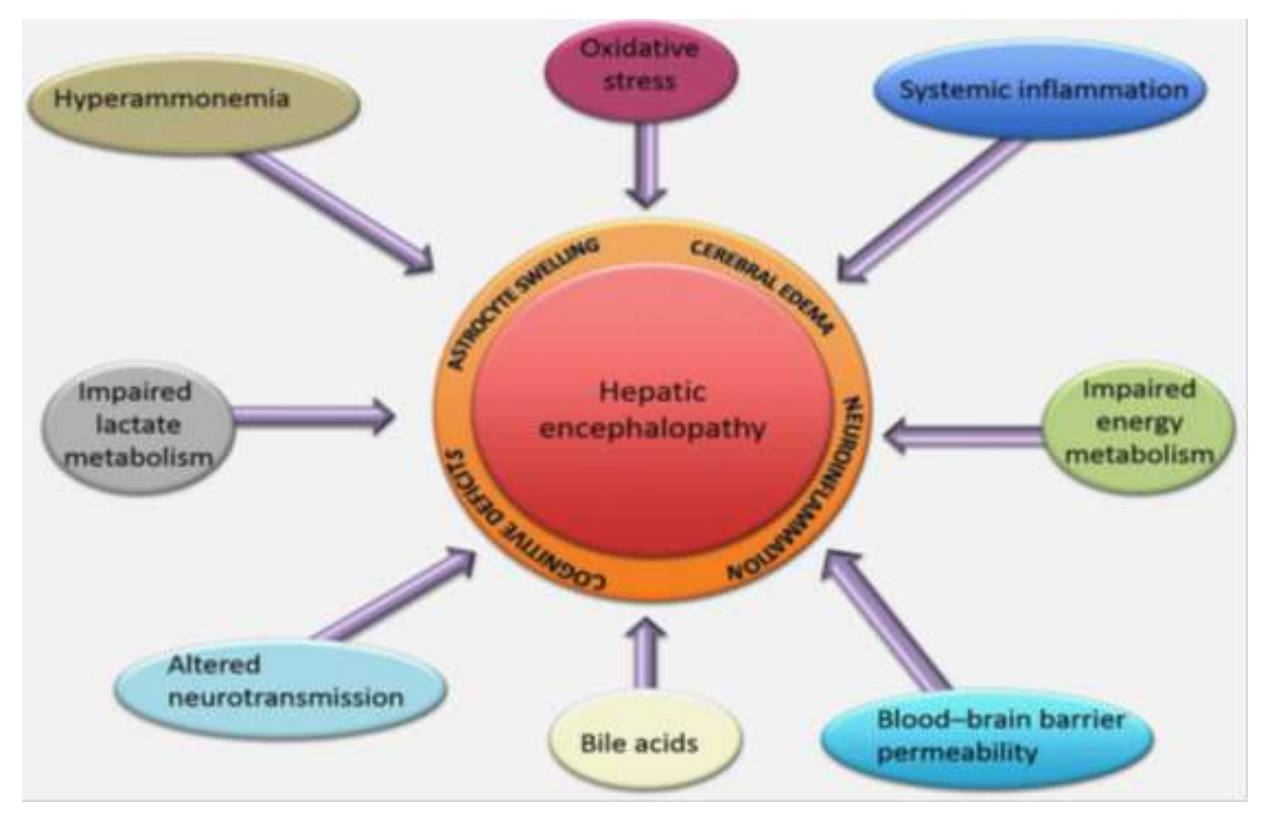

Figure 2. The etiology of hepatic encephalopathy [11].

High levels of chemokines pro-inflammatory has been detected in neurons, and imbalance between pro and anti inflammation signals acting on microglia receptors, Recently, sepsis encephalopathy lead to the swelling of inflammatory TNF and cytokines IL6, IL1 which impaired the permeability of endothelial cells it is very likely that bile acids related to defect blood brain barrier permeability [11], that jaundice associated with bile acids and lipids metabolism [12]. Oxidative stress, hypertrophy of fat cells and inflammation which increases fat absorption from blood circulation [13]. Therefore the study of disease cirrhosis a etiologies helps to diagnose hepatic encephalopathy progression [14]. Malnutrition has a significant role in the progression of the disease as protein deficiency is seen in patients [15]. There was a significant increase in anemia as well as bilirubin for many cases of disease [16]. Electroencephalogram analysis and critical flicker frequency as well as psychometric measures is useful to detect hepatic encephalopathy [17] Clinical characterization of minimal-HE diagnoses includes decrease concentration of sodium, thiamine, intracranial hemorrhage and sepsis [18] The cognitive abilities of the patient must also be examined with increased metabolic impairment [19], however there is still no scientific medical imaging to accurately diagnose hepatic encephalopathy [20]. It must distinguish between different brain diseases and hepatic encephalopathy, so psychological measurements should be confirmed especially in convulsions that associated with liver disease [21]. Figure 3 illustrates the ammonia metabolism in both the muscles, kidneys, liver and brain [22]. 


\section{Al-Nahrain Journal of Science}

ANJS, Vol.23 (1), March, 2020, pp. 7-12

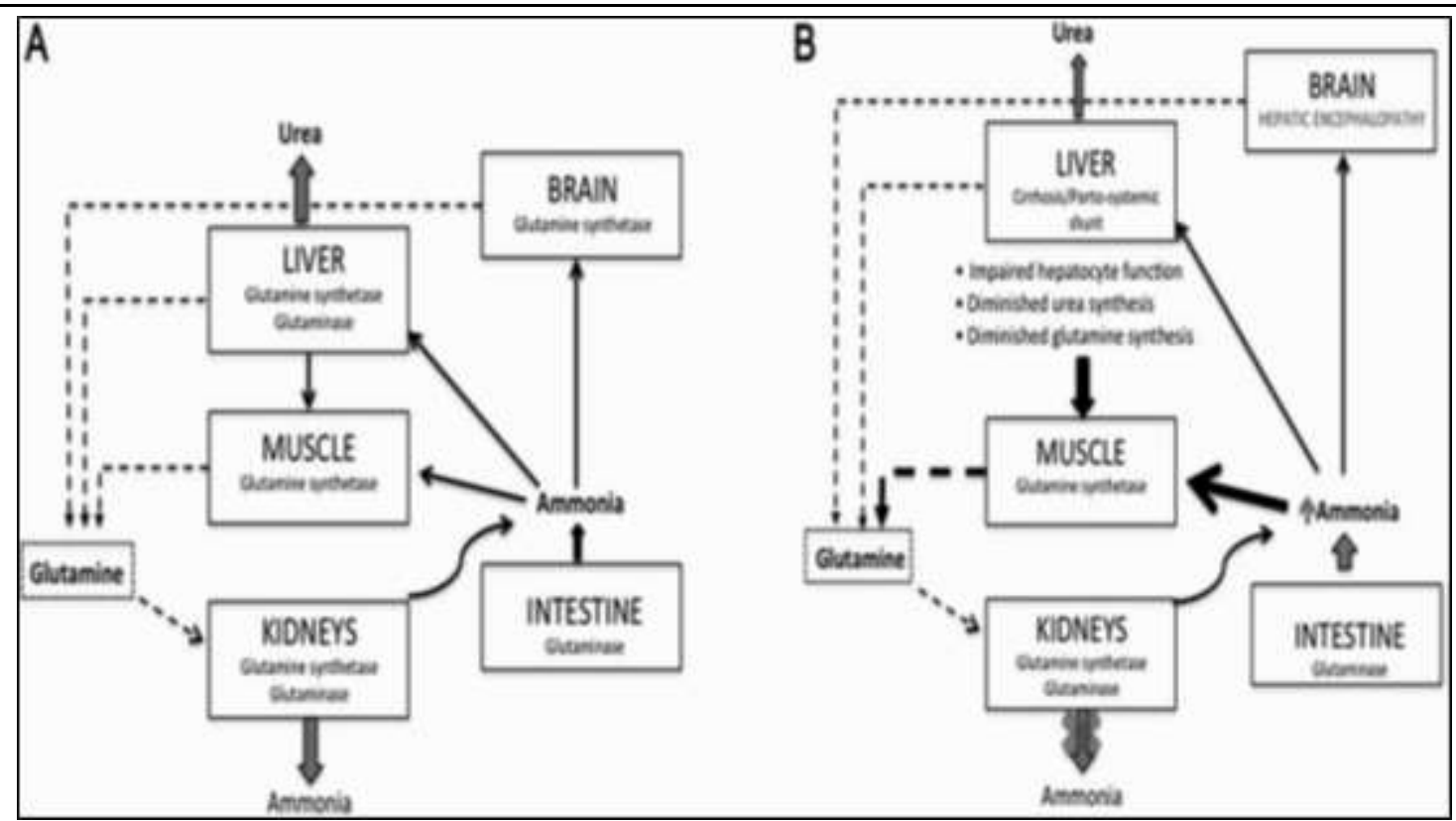

Figure 3. Illustrates the ammonia metabolism in both the muscles, kidneys, liver and brain. A. healthy subjects B. patients [22].

Myasthenia is associated with cirrhosis such as primary cholangitis, hepatitis and primary sclerosing cholangitis [23, 24]. Myasthenia is muscular weakness that has reduced physical activity, especially in adults started from ocular to the bulbar and skeletal muscles [25]. Its result from high levels of myostatin and ammonia accumulation in the blood where increased significant of myostatin in patients with cirrhosis especially in large skeletal muscles and deficiency of glutaminase synthetase that converts ammonia to glutamine as shown in figure 4 [26]. The liver's ability is impaired to remove hyperammonemia through urea cycle and therefore depends on the muscle to detoxify ammonia in patient with hepatic encephalopathy. Hyperammonemia induced brain absorption of aromatic amino acids such as tryptophan as a neurotransmitter of serotonin, which in turn leads to altered synthesis and to reduce neurological sensitization with increased C-reactive protein in bloodstream [27]. Antibodies against acetylcholine receptors and muscle kinase produce by $\mathrm{B}$ cells and $\mathrm{T}$ cells specifically the CD4+ helper cells is the main reasons for progressing myasthenia therefore acetyl cholinesterase inhibitors as a primary linear treatment of mild myasthenia by down degradation of acetylcholine which increases its flow to the nerve muscles

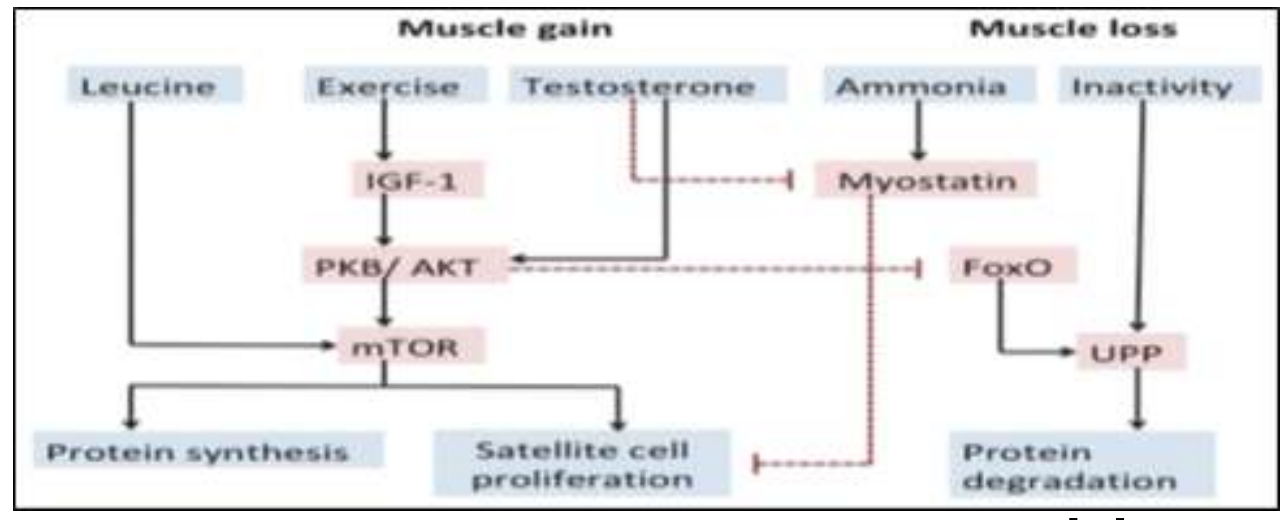

Figure 4. Regulated muscle and therapy possibilities [26] 


\title{
Al-Nahrain Journal of Science
}

\author{
ANJS, Vol.23 (1), March, 2020, pp. 7-12
}

Continuous arrows as activated pathways. Discontinuous lines as inhibitory pathways. IGF-1, insulin-like growth factor 1; PKB/AKT, protein kinase B; mTOR, mammalian target of rapamycin; FoxO, forkhead box transcription factor; UPP, ubiquitin-proteasome pathway. The neuromuscular junction a classic example of an antibody-mediated autoimmune disease [29]. Thymus gland playing role also to produce antibodies and differentiation of $\mathrm{T}$ lymphocytes that any disorder lead to increase anti acetylcholine receptors and developed Myasthenia[30]. In addition to that, lactulose, rifaximin or mixed can be treated [31]. It must regular use of convulsions drugs especially benzodiazepines where increased doses lead to hepatic encephalopathy glutamine synthase is also used to treat increased blood ammonia where astrocytes convert ammonia to glutamine but when accumulate in astrocytes leading to swelling and increase oxidative stress through free radical degradation in mitochondria [21, 32].

\section{Conclusions}

1. Hepatic encephalopathy is a central nervous system disorder due to liver failure.

2. Myasthenia is associated with hepatic encephalopathy that the liver's ability is impaired to remove hyperammonemia therefore depends on the muscle to detoxify ammonia.

3. A major factors are related to impaired lactate metabolism.

4. Rifaximin with lactulose as therapy for hepatic encephalopathy while lactulose overdose may lead to complications, such as dehydration, hyper-sodium and peritoneal skin irritation.

\section{References}

[1] Amodio, P.; "Hepatic encephalopathy: historical remarks"; J. Clin. Exp. Hepatol. 5(1), 4-6, 2015.

[2] Romero-Gómez, M.; Montagnese, S.; Jalan, R.; "Hepatic encephalopathy in patients with acute decompensation of cirrhosis and acuteon- chronic liver failure"; J. Hepatol. 62(2),437-47,2015.

[3] Thomsen, K.; Macnaughtan, J.; Tritto, G.; Mookerjee R.; Jalan, R.; "Clinical and pathophysiological characteristics of cirrhotic patients with grade 1 and minimal hepatic encephalopathy'; PLoS ONE 11(1), e0146076, 2016.
[4] Aldridge, D.; Tranah, E.; Shawcross, D.;

"Pathogenesis of hepatic encephalopathy: role of ammonia and systemic inflammation"; J. Clin. Exp. Hepatol. 5(1), S7-20, 2015.

[5] Wijdicks, E.; "Hepatic encephalopathy"; N. Engl. J. Med. 375(17),1660-70, 2016.

[6] Rackayova, V.; Braissant, O.; McLin, V.; Berset, C.; Lanz, B.; Cudalbu, C.; " $1 \mathrm{H}$ and $31 \mathrm{P}$ magnetic resonance spectroscopy in a rat model of chronic hepatic encephalopathy: in vivo longitudinal measurements of brain energy metabolism"; Metab. Brain Dis. 31(6), 1303-14, 2016.

[7] Anna, H.; Natalia, A.; Mohammed, S.; Rajiv, J.; "Hepatic encephalopathy: a critical current review"; Hepatol. Int. 12(1), 135-147, 2016.

[8] Olivia, T.; Joshua, M.; Nicola, A.; Mary, M.; Novraj, S.; Simon, D.; "Diagnosing and treating hepatic encephalopathyBritish" Journal of Hospital Medicine 76(11), 646-654, 2015.

[9] McMillin, M.; Frampton, G.; Seiwell, A.; Patel, N.; Jacobs, A.; DeMorrow, S.; "TGFB1 exacerbates bloodbrain barrier permeability in a mouse model of hepatic encephalopathy via upregulation of MMP9 and downregulation of claudin-5. Lab Invest"; PubMed 95(8), 903-13, 2015.

[10] Sawhney, R.; Jalan, R.; "Liver: the gut is a key target of therapy in hepatic encephalopathy"; Nat. Rev. Gastroenterol. Hepatol. 12(1), 7-8, 2015.

[11] Victoria, L.;Gurkarminder, S.; Sharon, D.; "Recent advances in hepatic"; F1000 Research 6(7),1637, 2017.

[12] Qutaiba, S.; Marwa, H.; "Influence of Thyroid Stimulating Hormone on Liver Enzymes Levels in Serum of Thyroid Disorder Iraqi Patients"; $\mathrm{Al}^{-}$ Nahrain J. Sci. 22 (3), 50-55, 2019.

[13] Faraj, R.; Jawad, A.; Badr, A.; "Effect of Body Mass Index on Abnormal Ovarian Secretion Hormones among Iraqi Women with Polycystic Ovarian Syndrome (PCOS)"; Al-Nahrain J. Sci. 22 (1), 40-45, 2019.

[14] Herbert, D.; "Hepatic Encephalopathy In Liver Cirrhosis",; J. Trans. Inter. Med. 5(1), 64-67, 2017.

[15] Peter F.; "Hepatic encephalopathy"; Gastroenterology Report 5(2), 138-147, 2017.

[16] Herbert, D.; "Hepatic Encephalopathy In Patients In Lviv (Ukraine)"; J. Trans. Inter. Med. 6(3), 146-151, 2018.

[17] Morgan, M.; Amodio, P.; Cook, N.; Jackson, C.; Kircheis, G.; Lauridsen, M.; Montagnese, S.; Schiff, S.; Weissenborn, K.; "Qualifying and quantifying minimal hepatic encephalopathy"; Metab. Brain Dis. 31(6), 1217-1229, 2016.

[18] Weissenborn, K.; "Challenges in diagnosing hepatic encephalopathy"; Neurochem. Res. 40(2), 265-73, 2015.

[19] Montagnese, S.; De-Rui, M.; Angeli, P.; Amodio, P.; "Neuropsychiatric performance in patients with cirrhosis: Who is "normal?"; J. Hepatol. 66(4), 825-35, 2017. 


\section{Al-Nahrain Journal of Science}

ANJS, Vol.23 (1), March, 2020, pp. 7-12

[20] Chen, H.; Chen, R.; Yang, M.; Teng, G.; Herskovits, E.; "Identification of minimal hepatic encephalopathy in patients with cirrhosis based on white matter imaging and Bayesian data mining"; AJNR Am. J. Neuroradiol 36(3), 481-7, 2015.

[21] Aneesa, R.; Chowdhury, N.; Marcus, S.; "Disorder Exacerbated by Hepatic Encephalopathy: A Case Report” Open Access Macedonian Journal of Medical 7(10), 1669-1671, 2019.

[22] Anna, H.; Rajiv, J.; "Hepatic Encephalopathy: New Treatments", Clinical Liver Disease 5(5), 109-111, 2015.

[23] Madiha, M.; Habiba, M.; Sami, T.; Adel, K.; "Autoimmune hepatitis-primary biliary cirrhosis: Overlap syndrome concomitant with unexpected myasthenia and thymoma”; Open J. Clin. Diagn. 5(1), 20-3, 2015.

[24] Lindor, K.; Bowlus, C.; Boyer, J.; Levy, C.; Mayo, M.; "Primary biliary cholangitis: 2018 practice guidance from the American Association for the Study of Liver Diseases"; Hepatology 69(1), 394-419, 2019.

[25] Slater, C.; "The structure of human neuromuscular junctions: some unanswered molecular questions"; Int. J. Mol. Sci. 18(10), 2183, 2017.

[26] Ankur, J.; Rakesh, K.; "Sarcopenia:Ammonia metabolism and hepatic Encephalopathy" Int. J. Gen. Med. 7, 1-10, 2019.
[27] Vijay, P.; Joshua, M.; Nicolas, M.; Mary, M.; Nicola, A.; Simon, D.; "The why and wherefore of hepatic encephalopathy"; Int. J. Gen. Med. 8, 381-390, 2015.

[28] Sudhir, V.; Sriganesh, K.; "Management of Myasthenia Gravis"; J. Neuroanaesthesiol Crit. Care 6(2),153-159, 2019.

[29] Inga, K.; Ruth, H.; Myasthenia, G.; "Pathogenic Effects of Autoantibodies on Neuromuscular Architecture Cells" 8, 671, 2019.

[30] Gautam, R.; "Overlap Syndrome and Myasthenia Gravis" 2019.

[31] Nardelli, S.; Ridola, L.; Gioia, S.; Riggio, O.; "Management of Hepatic Encephalopathy NotResponsive to First-Line Treatments"; Curr. Treat. Options Gastroenterol 16(2), 253-259, 2018.

[32] Nardelli, S.; Allampati, S.; Riggio, O.; Mullen, K.; Prakash, R.; Gioia, S.; Unser, A.; White, M.; Fagan, A.; Wade, J.; Farcomeni, A.; Gavis, E.; Bajaj, J.; "Hepatic Encephalopathy Associated with Persistent Learning Impairments Despite Adequate Medical Treatment: A Multicenter", Int. Study. Dig. Dis. Sci. 62, 794-800, 2017. 